\title{
Phenotypic, additive genetic and environment correlations of maize landraces populations in family farm systems
}

\author{
Luciane Bertoletti Barros ${ }^{1}$ Rosângela Maria Pinto Moreira²; Josué Maldonado Ferreira ${ }^{2 *}$ \\ ${ }^{1}$ UEL - Programa de Pós-Graduação em Genética e Biologia Molecular, C.P. 6001 - 86051-990 - Londrina, PR - \\ Brasil. \\ ${ }^{2}$ UEL/Centro de Ciências Biológicas, Depto. de Biologia Geral, Lab. de Melhoramento Genético de Plantas, C.P. \\ 6001 - 86051-990 - Londrina, PR - Brasil. \\ *Corresponding author <josuemf@uel.br>
}

\begin{abstract}
Knowledge of the association between characters is very important in the genetic breeding programs, but there is limited information about correlations between traits in maize landraces in the current literature. The objectives of this study were to estimate phenotypic, additive genetic and environment correlations among traits in maize landraces, which were cultivated in family farm systems, in order to guide the participatory breeding on maize landraces. Between 294 and 400 half-sib progenies from the six populations were evaluated, subdivided in triple 7X7 and 10X10 lattice design, respectively, with single row plots of $4 \mathrm{~m}$ long and spaced $1.00 \times 0.20 \mathrm{~m}$. Estimates of correlation coefficients are reported for nine traits: grain yield $(\mathrm{GY})$, relation grain weight per ear weight $(\mathrm{GE})$, ears per plant (EP), days to flower (DF), plant height $(\mathrm{PH})$, ear height $(\mathrm{EH})$, percentage of root loding $(\% \mathrm{~L})$, percentage of stalk loding $(\% \mathrm{BS})$ and percentage of damaged ears $(\% \mathrm{DE})$. A high number of additive genetic correlation $\left(\mathrm{r}_{\mathrm{A}}\right)$ was obtained between grain yield and the other traits, in maize landraces populations, cycles and locations. For grain yield, the highest averages of the $r_{A}$ estimate were with EP (0.67), GE (0.47) and \%DE (-0.63). In contrast to the current published researches, negative correlations between grain yield and days to flower were observed.
\end{abstract}

Key words: Zea mays L., population, recurrent selection, participatory breeding

\section{Correlações fenotípicas, genéticas aditivas e ambientais de populações de milho crioulo em sistemas de agricultura familiar}

\begin{abstract}
RESUMO: O conhecimento da associação entre caracteres é de grande importância nos programas de melhoramento genético, contudo a literatura apresenta limitadas informações sobre correlações em populações de milho crioulo. Os objetivos deste estudo foram estimar correlações fenotípicas, genéticas aditivas e ambientais entre caracteres de populações de milho crioulo, cultivados em sistemas de agricultura familiar, para orientar o programa de melhoramento genético participativo. Entre 294 e 400 de progênies de meios-irmãos de seis populações, foram avaliadas em látices triplos $7 \mathrm{X} 7$ e 10X10, respectivamente, com parcelas de $4 \mathrm{~m}$ de comprimento e espaçadas $1,00 \times 0,20 \mathrm{~m}$. Os coeficientes de correlações foram estimados para nove características: produtividade de grãos total (GY), relação peso de grãos por peso de espiga (GE), $\mathrm{n}^{\circ}$ de espigas por planta (EP), $\mathrm{n}^{\mathrm{o}}$ de dias do florescimento $(\mathrm{DF})$, altura da planta $(\mathrm{PH})$, altura espiga $(\mathrm{EH})$, porcentagem de acamamento $(\% \mathrm{~L})$, porcentagem de colmos quebrados (\%BS) e porcentagem de espigas danificadas (\%DE). Houve um alto número de correlação genética aditiva $\left(\mathrm{r}_{\mathrm{A}}\right)$ entre produtividade de grãos e as demais características, nas populações de milho crioulo, ciclos e locais. As maiores médias das estimativas $\mathrm{r}_{\mathrm{A}}$ para o produtividade de grãos foram com EP $(0,67)$, GE $(0,47)$ e DE\% $(-0,63)$. Diferentemente dos trabalhos publicados, foram observadas correlações negativas entre produtividade de grãos e dias para florescimento.
\end{abstract}

Palavras-chave: Zea mays, população, seleção recorrente, melhoramento genético participativo

\section{Introduction}

Brazilian maize landraces populations were originated through Amerindian races, introduced from central and of North America and several commercial materials (Paterniani, 1998; Paterniani et al., 2000; Sánchez et al., 2007). These varieties have been broadly and independently cultivated throughout Brazilian regions and they have relevant socio-economic importance for the family farm systems. As a result, accessions were developed and selected for different environments and morphological characteristics (Paterniani et al., 2000;
Warburton et al., 2008; Wietholter et al., 2008). However, more consistent agronomic and genetic knowledge about these collections is still lacking and it is a serious limitation to utilizing, managing, and conserving of maize landrace gene pools (Nass et al., 1993; Andrade et al., 2002; Teixeira et al., 2002).

The use of genotypic correlation helps evaluating the magnitude and direction of associations between characters facilitating the application of indirect selection, because genetic change in a given trait may change other traits, leading to faster and larger genetic gains in maize breeding programs (Vencovsky and Barriga, 1992). There-

Sci. Agric. (Piracicaba, Braz.), v.67, n.6, p.685-691, November/December 2010 
fore, the selection for another trait may result in indirect response in the low heritable trait, provided the following conditions are satisfied: the genetic correlation between them is substantial, and the heritability of the secondary trait is greater than that of the primary trait (Hallauer and Miranda Filho, 1995). Many authors have estimated genetic correlations among different maize traits in different maize types (Lemos et al., 1992; González et al., 1994; Pinto et al., 2000; Silva et al., 2001; Farias Neto and Miranda Filho, 2001; Saleh et al., 2002; Granate et al., 2002; Daros et al., 2004; Santos et al., 2005; Andrade and Miranda Filho, 2008). However, there is limited published research with genetic correlation estimates for maize landraces. Thus, the objectives of this study were to estimate phenotypic, genotypic and environment correlations among traits in maize landraces, that were cultivated in family farm systems.

\section{Material and Methods}

Six maize landraces populations, four with yellow endosperm (Caiano, Carioca, Macaco and Palha Roxa) and two with white endosperm (Cinquentinha and Maizena), that have been improved by the Participatory Breeding Program of Maize Landrace (Ferreira et al., 2006), were used in this study. These landraces were selected individually, through two or three cycles of halfsib family selection, using selection intensity at about $12.0 \%$ to $16.3 \%$, according to the methodology showed by Hallauer and Miranda Filho (1995), in family farm systems.

The populations were evaluated with 300 and 400 half-sib progenies in three and four 10X10 lattice experiments, respectively, with three replications each, during the initial cycle of recurrent selection (CO). In the following cycles of the recurrent selection (C1 and C2) 294 half-sib progenies in six 7X7 lattice experiments with three replications (Table 1) were evaluated. Experimental plots were represented by a single row $4 \mathrm{~m}$ long, spaced $1 \mathrm{~m}$ apart, with 20 plants after thinning. The following traits were taken for analysis: GY - total grain yield corrected for $13.5 \%$ moisture (g per plant and $\mathrm{t} \mathrm{ha}^{-1}$ ); GE relation grain weight per ear weight; EP - ears per plant; DF - days to flower, (average between days to silk extrusion and days to anthesis); $\mathrm{PH}$ - plant height $(\mathrm{cm}), \mathrm{EH}$ - ear height $(\mathrm{cm}) ; \% \mathrm{~L}$ - percentage of root loding; \%BS percentage of stalk loding; and \%DE - percentage of damaged ears.

The half-sib progenies were obtained and evaluated in farms of Paraná and Santa Catarina states, Brazil, using organic systems of production. The climate of these local is subtropical humid (Table 1).

The basic statistical model for variance and covariance analyses following the lattice design is $\mathrm{Y}_{\mathrm{ik} / \mathrm{j}}=\mathrm{m}+$ $\mathrm{p}_{\mathrm{i}}+\mathrm{r}_{\mathrm{j}}+(\mathrm{b} \mid \mathrm{r})_{\mathrm{k}(\mathrm{i})}+\mathrm{e}_{\mathrm{ik} / \mathrm{i}_{\mathrm{i}}}$ where $\mathrm{Y}_{\mathrm{ik} / \mathrm{i}}$ is the observed mean of $\mathrm{i}^{\text {th }}$ progeny in the $\mathrm{k}^{\text {th }}$ block of the $\mathrm{j}^{\text {th }}$ replication; $\mathrm{m}$ is the general mean; $p_{i}$ is the random effect of the $i^{\text {th }}$ progeny; $r_{j}$ is the random effect of the $j^{\text {th }}$ replication; $(b \mid r)_{k(i)}$ is the random effect of $\mathrm{k}^{\text {th }}$ block of the $\mathrm{j}^{\text {th }}$ replication; and $e_{i k / j}$ is the error term. The individual variance and covariance analyses were grouped to compute the following estimates for each population, local and cycle of selection: environmental variance $\hat{\sigma}_{\mathrm{E}}^{2}=\mathrm{M}_{\mathrm{E}}$; phenotypic variance of progenies means within experiments

$\hat{\sigma}_{\mathrm{F}}^{2}=\left[\frac{(\mathrm{k}+1)}{\mathrm{k}}\right]\left[\frac{\left(\mathrm{M}_{\mathrm{P} / \mathrm{Exp}}\right)}{\mathrm{r}}\right] ;$

additive genetic variance within experiments

$\hat{\sigma}_{\mathrm{A}}^{2}=4\left[\frac{(\mathrm{k}+1)}{\mathrm{k}}\right]\left[\frac{\left(\mathrm{M}_{\mathrm{P} / \mathrm{Exp}}-\mathrm{M}_{\mathrm{E}}\right)}{\mathrm{r}}\right] ;$

environmental covariance CÔ $\mathrm{V}_{\mathrm{Ex} \cdot \mathrm{y}}=\mathrm{P}_{\mathrm{F}}$; phenotypic covariance of progenies means within experiments

$\mathrm{CÔV}_{\mathrm{F} \times \mathrm{Y}}=\left[\frac{(\mathrm{k}+1)}{\mathrm{k}}\right]\left[\frac{\left(\mathrm{P}_{\mathrm{E} / \mathrm{Exp}}\right)}{\mathrm{r}}\right]$;

genetic covariance among progenies within experiments

CÔV $\mathrm{V}_{\mathrm{Axy}}=4\left[\frac{(\mathrm{k}+1)}{\mathrm{k}}\right]\left[\frac{\left(\mathrm{P}_{\mathrm{P} / \mathrm{Exp}}-\mathrm{P}_{\mathrm{E}}\right)}{\mathrm{r}}\right]$;

environmental correlation coefficient $r_{\mathrm{E}}=\frac{C \hat{O} V_{\mathrm{Exy}}}{\hat{\sigma}_{\mathrm{Ex}} \cdot \hat{\sigma}_{\mathrm{E} y}}$;

phenotypic (means) correlation coefficient $r_{\mathrm{F}}=\frac{C O \hat{V} V_{\mathrm{Fx} \cdot \mathrm{y}}}{\hat{\sigma}_{\mathrm{Fx}} \cdot \hat{\sigma}_{\mathrm{Fy}}}$; additive correlation coefficient $r_{A}=\frac{C \hat{O} V_{A x . y}}{\hat{\sigma}_{A x} \cdot \hat{\sigma}_{A y}}$;

where: $\mathrm{M}_{\mathrm{E}}$ is the mean squares of error; $\mathrm{M}_{\mathrm{P} / \mathrm{Exp}}$ is the mean squares of progeny within experiments; $P_{E}$ is the mean products of error; $\mathrm{P}_{\mathrm{P} / \mathrm{Exp}}$ is the mean products of progeny within experiments. $\mathrm{X}$ and $\mathrm{Y}$ refer to pairs of traits (Viana and Regazzi, 1999; Silva et al, 2000).

The Student's $t$ test was used to determinate the significance of the phenotypic, additive and environmental correlation coefficients at the $5 \%$ and $1 \%$ probability levels.

\section{Results and Discussion}

A progeny variation $(p<0.01)$ was detected for total grain yield (GY), days to flower (DF), plant height $(\mathrm{PH})$, ear height $(\mathrm{EH})$ in all experiments. The others traits had significant progeny effects in the majority of experiments. So, there is genetic variability for the populations in the different cycles and local for the vast majority of evaluated traits (Table 2).

The maximum and minimum population average by local and cycles of selection, were, respectively, 8.18 and $1.86 \mathrm{t} \mathrm{ha}^{-1}$ for grain yield; 0.85 and 0.62 for relation grain weight per ear weight; 1.12 and 0.62 ears per plant; $50.3 \%$ and $5.7 \%$ for percentage of damaged ears; 111 and 80 for days to flower; 309 and $171 \mathrm{~cm}$ for plant height; 197 and $85 \mathrm{~cm}$ for ear height; $24.3 \%$ and $0 \%$ for percentage of root lodging; and, $53.7 \%$ and $0.8 \%$ percentage of stalk lodging (Table 2). These results show a good perfor- 
mance of populations in family farm systems for the majority of locals and cycles of selection, when compared to the average of $5.35 \mathrm{t} \mathrm{ha}^{-1}$ (density of 50,000 plants $\mathrm{ha}^{-1}$ ) mean estimates for Brazilian populations (Miranda Filho, 1985), particularly when the rustic environmental conditions in which those experiments were conducted are considered. Poor performance for grain yield and other traits were observed in few locations.

The majority of the traits, evaluated in the cycles and local, exhibited additive correlation coefficients $(p<0.01$ or $p<0.05)$ with grain yield. High additive correlations were frequently observed between grain yield and the traits: grain weight per ear weight (GE), ears per plant (EP) and percentage of damaged ears (\%DE). High correlations between yield and ears per plant have also been reportedby Lemos et al. (1992),
González et al. (1994) and Silva et al. (2001). For the traits days to flower $(\mathrm{DF})$, plant height $(\mathrm{PH})$, ear height $(\mathrm{EH})$, percentage of root loding $(\% \mathrm{~L})$, percentage of stalk loding (\%BS), smaller correlation with grain yield were predominant, but significant for the majority. The high degrees of freedom included in the t test must have contributed to show significant correlation in low estimates. The additive correlation indicated that an increase of grain yield of these maize landraces in family farm systems, is associated with increase of grain weight per ear weight (GE), number of ears per plant (EP) and decrease for percentage of damaged ears (\%DE) (Table 3).

For plant height and ear height, frequently, the significant additive correlations with grain yield were positive, ranging from 0.58 to -0.21 and 0.63 to -0.21 , and only three cases had low negative estimates (Table 3 ).

Table 1 - Landraces populations (Land), cycles (C) and local (L) of selection, counties with locations, lattice types, numbers of experiment (NE) and sowing dates.

\begin{tabular}{|c|c|c|c|c|c|c|c|c|}
\hline Land & $\mathrm{C} / \mathrm{L}$ & County & Lat. $\left({ }^{\circ}\right)$ & Long. $\left(^{\circ}\right)$ & Altitude & Lattice & $\mathrm{NE}$ & Sowing date \\
\hline \multirow{6}{*}{ Caiano } & COL1 & Palmeira & $25^{\circ} 25^{\prime} \mathrm{S}$ & $50^{\circ} 00^{\prime}$ & $864 \mathrm{~m}$ & $10 \times 10$ & 4 & $11 / 21 / 2000$ \\
\hline & COL2 & Bituruna & $26^{\circ} 10^{\prime} \mathrm{S}$ & $51^{\circ} 34^{\prime}$ & $914 \mathrm{~m}$ & $10 \times 10$ & 4 & $11 / 11 / 2000$ \\
\hline & C1L1 & Palmeira & $25^{\circ} 25^{\prime} \mathrm{S}$ & $50^{\circ} 00^{\prime}$ & $864 \mathrm{~m}$ & $7 \times 7$ & 6 & $10 / 22 / 2002$ \\
\hline & C1L2 & Cruz Machado & $26^{\circ} 01^{\prime} \mathrm{S}$ & $51^{\circ} 21^{\prime}$ & $940 \mathrm{~m}$ & $7 \times 7$ & 6 & $10 / 07 / 2002$ \\
\hline & $\mathrm{C} 2 \mathrm{~L} 1$ & Palmeira & $25^{\circ} 25^{\prime} \mathrm{S}$ & $50^{\circ} 00^{\prime}$ & $864 \mathrm{~m}$ & $7 \times 7$ & 6 & $10 / 25 / 2004$ \\
\hline & C2L2 & Cruz Machado & $26^{\circ} 01^{\prime} \mathrm{S}$ & $51^{\circ} 21^{\prime}$ & $940 \mathrm{~m}$ & $7 \times 7$ & 6 & $10 / 09 / 2004$ \\
\hline \multirow{6}{*}{ Carioca } & COL1 & São Mateus do Sul & $25^{\circ} 52^{\prime} \mathrm{S}$ & $50^{\circ} 22^{\prime}$ & $835 \mathrm{~m}$ & $10 \times 10$ & 4 & $10 / 29 / 2000$ \\
\hline & COL2 & Bituruna & $26^{\circ} 10^{\prime} \mathrm{S}$ & $51^{\circ} 34^{\prime}$ & $914 \mathrm{~m}$ & $10 \times 10$ & 3 & $10 / 31 / 2000$ \\
\hline & C1L1 & São Mateus do Sul & $25^{\circ} 52^{\prime} \mathrm{S}$ & $50^{\circ} 22^{\prime}$ & $835 \mathrm{~m}$ & $7 \times 7$ & 6 & $10 / 13 / 2002$ \\
\hline & C1L2 & Bituruna & $26^{\circ} 10^{\prime} \mathrm{S}$ & $51^{\circ} 34^{\prime}$ & $914 \mathrm{~m}$ & $7 \times 7$ & 6 & $10 / 18 / 2002$ \\
\hline & C2L1 & São Mateus do Sul & $25^{\circ} 52^{\prime} \mathrm{S}$ & $50^{\circ} 22^{\prime}$ & $835 \mathrm{~m}$ & $7 \times 7$ & 6 & $10 / 19 / 2004$ \\
\hline & C2L2 & Bituruna & $26^{\circ} 10^{\prime} \mathrm{S}$ & $51^{\circ} 34^{\prime}$ & $914 \mathrm{~m}$ & $7 \times 7$ & 6 & $10 / 29 / 2004$ \\
\hline \multirow{5}{*}{ Palha Roxa } & COL1 & Palmeira & $25^{\circ} 25^{\prime} \mathrm{S}$ & $50^{\circ} 00^{\prime}$ & $864 \mathrm{~m}$ & $10 \times 10$ & 4 & $10 / 20 / 2000$ \\
\hline & COL2 & Bituruna & $26^{\circ} 10^{\prime} \mathrm{S}$ & $51^{\circ} 34^{\prime}$ & $914 \mathrm{~m}$ & $10 \times 10$ & 4 & $10 / 26 / 2000$ \\
\hline & C1L1 & Palmeira & $25^{\circ} 25^{\prime} \mathrm{S}$ & $50^{\circ} 00^{\prime}$ & $864 \mathrm{~m}$ & $7 \times 7$ & 6 & $10 / 15 / 2002$ \\
\hline & C1L2 & Bituruna & $26^{\circ} 10^{\prime} \mathrm{S}$ & $51^{\circ} 34^{\prime}$ & $914 \mathrm{~m}$ & $7 \times 7$ & 6 & $10 / 05 / 2002$ \\
\hline & C2L1 & Palmeira & $25^{\circ} 25^{\prime} \mathrm{S}$ & $50^{\circ} 00^{\prime}$ & $864 \mathrm{~m}$ & $7 \times 7$ & 6 & $10 / 06 / 2004$ \\
\hline \multirow{5}{*}{ Macaco } & COL1 & Palmeira & $25^{\circ} 25^{\prime} \mathrm{S}$ & $50^{\circ} 00^{\prime}$ & $864 \mathrm{~m}$ & $10 \times 10$ & 4 & $10 / 09 / 2000$ \\
\hline & COL2 & Irineópolis & $26^{\circ} 14^{\prime} \mathrm{S}$ & $50^{\circ} 48^{\prime}$ & $762 \mathrm{~m}$ & $10 \times 10$ & 4 & $11 / 09 / 2000$ \\
\hline & C1L1 & Palmeira & $25^{\circ} 25^{\prime} \mathrm{S}$ & $50^{\circ} 00^{\prime}$ & $864 \mathrm{~m}$ & $7 \times 7$ & 6 & $10 / 10 / 2002$ \\
\hline & C2L1 & Palmeira & $25^{\circ} 25^{\prime} \mathrm{S}$ & $50^{\circ} 00^{\prime}$ & $864 \mathrm{~m}$ & $7 \times 7$ & 6 & $10 / 21 / 2004$ \\
\hline & C2L2 & Irineópolis & $26^{\circ} 14^{\prime} \mathrm{S}$ & $50^{\circ} 48^{\prime}$ & $762 \mathrm{~m}$ & $7 \times 7$ & 6 & $10 / 07 / 2004$ \\
\hline \multirow{4}{*}{ Maisena } & COL1 & Rebouça & $25^{\circ} 37^{\prime} \mathrm{S}$ & $50^{\circ} 41^{\prime}$ & $815 \mathrm{~m}$ & $10 \times 10$ & 3 & $10 / 23 / 2001$ \\
\hline & COL2 & Bituruna & $26^{\circ} 10^{\prime} \mathrm{S}$ & $51^{\circ} 34^{\prime}$ & $914 \mathrm{~m}$ & $10 \times 10$ & 3 & $10 / 26 / 2001$ \\
\hline & C1L1 & Irati & $25^{\circ} 28^{\prime} \mathrm{S}$ & $50^{\circ} 39^{\prime}$ & $820 \mathrm{~m}$ & $7 \times 7$ & 6 & $10 / 24 / 2003$ \\
\hline & C1L2 & Bituruna & $26^{\circ} 10^{\prime} \mathrm{S}$ & $51^{\circ} 34^{\prime}$ & $914 \mathrm{~m}$ & $7 \times 7$ & 6 & $10 / 30 / 2003$ \\
\hline \multirow{4}{*}{ Cinquentinha } & C1L1 & Fernandes Pinheiro & $25^{\circ} 25^{\prime} \mathrm{S}$ & $50^{\circ} 33^{\prime}$ & $824 \mathrm{~m}$ & $7 \times 7$ & 6 & $10 / 09 / 2002$ \\
\hline & C1L2 & Bituruna & $26^{\circ} 10^{\prime} \mathrm{S}$ & $51^{\circ} 34^{\prime}$ & $914 \mathrm{~m}$ & $7 \times 7$ & 6 & $10 / 19 / 2002$ \\
\hline & C2L1 & Fernandes Pinheiro & $25^{\circ} 25^{\prime} \mathrm{S}$ & $50^{\circ} 33^{\prime}$ & $824 \mathrm{~m}$ & $7 \times 7$ & 6 & $10 / 15 / 2004$ \\
\hline & C2L2 & Bituruna & $26^{\circ} 10^{\prime} \mathrm{S}$ & $51^{\circ} 34^{\prime}$ & $914 \mathrm{~m}$ & $7 \times 7$ & 6 & $11 / 04 / 2004$ \\
\hline
\end{tabular}


Table 2 - Means and significance of mean squares of progeny within experiments for nine traits ${ }^{1}$ in different cycles (C) and local (L).

\begin{tabular}{|c|c|c|c|c|c|c|c|c|c|c|}
\hline Land & $\mathrm{C} / \mathrm{L}$ & GY & GE & EP & $\% \mathrm{DE}$ & DF & $\mathrm{PH}$ & $\mathrm{EH}$ & $\% \mathrm{~L}$ & $\% \mathrm{BS}$ \\
\hline \multirow{6}{*}{ Caiano } & COL1 & $4.29 \% *$ & $0.80 * *$ & $1.04 \% *$ & $13.1 \% *$ & $86.6 * *$ & $203 * *$ & $107 \% *$ & $9.1 * *$ & $8.2 \mathrm{~ns}$ \\
\hline & COL2 & $4.23^{* * \%}$ & $0.75 \%$ & $0.98 \% *$ & $30.7^{* * *}$ & $84.0 \% *$ & $253 \%$ & $144 \%$ & $0.9 * *$ & $2.2 \%$ \\
\hline & C1L1 & $7.92 * *$ & $0.82 * *$ & $0.99 * *$ & $9.3 * *$ & $86.4 * \%$ & $244 \% *$ & $143 *$ & $0.3 \mathrm{~ns}$ & $2.9^{*}$ \\
\hline & C1L2 & $3.61 \%$ & $0.80 \%$ & $0.90 \% *$ & $18.6 * *$ & $100.2 \%$ & $171 \%$ & $85 \%$ & $0.6 \mathrm{~ns}$ & $5.0 *$ \\
\hline & C2L1 & $7.75 \%$ & $0.80 \mathrm{~ns}$ & $1.10 \%$ & $15.7 *$ & $83.7 * \%$ & $258 * \%$ & $156 \%$ & $4.5 \mathrm{~ns}$ & $2.6 \%$ \\
\hline & C2L2 & $2.66 * *$ & $0.67 *$ & $0.92 \% *$ & $26.2 \% *$ & $110.5 \%$ & $177 \% *$ & $85^{\% *}$ & - & -- \\
\hline \multirow{6}{*}{ Carioca } & COL1 & $6.55 \%$ & $0.81 \%$ & $1.01 \% *$ & $21.8 *$ & $86.8^{* * *}$ & $300 \% *$ & $182 \%$ & $3.6 \%$ & $12.1 \% *$ \\
\hline & COL2 & $4.40 \% *$ & $0.78 *$ & $0.99 \mathrm{~ns}$ & $15.3 * *$ & $85.4 \%$ & $246 \%$ & $139 *$ & $4.1 \mathrm{~ns}$ & $3.9 \mathrm{~ns}$ \\
\hline & C1L1 & $6.11 \%$ & $0.85 \%$ & $0.92 \%$ & $22.6 * *$ & $92.2 * *$ & $253 \%$ & $143 \%$ & $3.6 \%$ & $7.1 \%$ \\
\hline & C1L2 & $4.17 \% *$ & $0.80 *$ & $0.88 \mathrm{~ns}$ & $7.0 \mathrm{~ns}$ & $90.5^{5 *}$ & $221 \%$ & $127 *$ & $3.7 \mathrm{~ns}$ & $6.4 \mathrm{~ns}$ \\
\hline & C2L1 & $4.42^{* * x}$ & $0.72 * *$ & $0.95^{* * *}$ & $32.2 * *$ & $88.7^{* * \%}$ & $276 * \%$ & $169 *$ & $0.0 \mathrm{~ns}$ & $53.7 * *$ \\
\hline & C2L2 & $1.86 * *$ & $0.62 \%$ & $0.88 \% *$ & $50.3 *$ & $96.1 * *$ & $246 \%$ & $144 \%$ & -- & - \\
\hline \multirow{5}{*}{ Palha Roxa } & CoL1 & $7.77 \%$ & $0.83 *$ & $1.05 \%$ & $12.0 * *$ & $86.4 \% *$ & $281 \%$ & $173 *$ & $2.6 * \%$ & $10.2 * *$ \\
\hline & COL2 & $4.25^{* *}$ & $0.78 \mathrm{~ns}$ & $0.97 \mathrm{~ns}$ & $20.4^{* \%}$ & $93.9 \%$ & $258 \%$ & $151^{* *}$ & $7.6 \% \%$ & $5.4 \% *$ \\
\hline & C1L1 & $8.18^{* * *}$ & $0.86 \%$ & $1.00 * *$ & $10.5 \mathrm{~ns}$ & $79.8 * *$ & $301 \%$ & $184 * *$ & $1.0 \mathrm{~ns}$ & $3.9 \mathrm{~ns}$ \\
\hline & C1L2 & $6.41 \%$ & $0.85 \%$ & $0.98 \mathrm{~ns}$ & $7.2 \mathrm{~ns}$ & $93.3 * \%$ & $253 \%$ & $145 \%$ & $1.3 \mathrm{~ns}$ & $1.7 \mathrm{~ns}$ \\
\hline & C2L1 & $6.24 \% *$ & $0.84 *$ & $0.97 \% *$ & $5.7 \mathrm{~ns}$ & $96.5^{* * *}$ & $255 \%$ & $174 * *$ & $0.1 \mathrm{~ns}$ & $0.8 * *$ \\
\hline \multirow{5}{*}{ Macaco } & COL1 & $7.03 \%$ & $0.83 \%$ & $0.99 \mathrm{~ns}$ & $13.9 *$ & $83.7 \% *$ & $263 \%$ & $154 \%$ & $7.1 \%$ & $7.5 \%$ \\
\hline & COL2 & $6.03 \%$ & $0.83 *$ & $0.93 \% *$ & $10.7 * *$ & $81.5 \%$ & $309 * *$ & $197 * *$ & $10.3 * *$ & $8.7 \mathrm{~ns}$ \\
\hline & C1L1 & $6.58 \%$ & $0.84 \%$ & $0.93 \%$ & $19.7 * *$ & $82.5 \%$ & $264 \%$ & $158 \%$ & $24.3 \%$ & $8.5^{\% *}$ \\
\hline & C2L1 & $6.08 \% *$ & $0.84 * *$ & $0.91 * *$ & $8.7 \mathrm{~ns}$ & $85.3 * *$ & $275 *$ & $172 *$ & $1.2 * *$ & $3.1 *$ \\
\hline & $\mathrm{C} 2 \mathrm{~L} 2$ & $2.48 \% *$ & $0.69 *$ & $0.62 * *$ & $48.2 * *$ & $101.4 \% *$ & $231 \%$ & $128 \%$ & $0.0 \mathrm{~ns}$ & $6.9 * *$ \\
\hline \multirow{4}{*}{ Maisena } & COL1 & $5.03 \%$ & $0.77 \mathrm{~ns}$ & $1.07 \mathrm{~ns}$ & $29.7 * *$ & $93.9 * \%$ & $287 \%$ & $183 \%$ & $7.0 \mathrm{~ns}$ & $18.5 \%$ \\
\hline & COL2 & $4.36 * *$ & $0.72 \mathrm{~ns}$ & $1.03 \mathrm{~ns}$ & $37.9 \mathrm{~ns}$ & $101.3 \% *$ & $265 \%$ & $164 \%$ & $4.5 \mathrm{~ns}$ & $16.6 \mathrm{~ns}$ \\
\hline & C1L1 & $3.31 \%$ & $0.79 *$ & $0.98 \% *$ & $45.2 \%$ & $96.4 \% *$ & $282 \%$ & $172 \%$ & $4.5 \%$ & $21.6 \%$ \\
\hline & C1L2 & $3.38 \%$ & $0.77 \mathrm{~ns}$ & $0.83 *$ & $43.7 \mathrm{~ns}$ & $101.4 \%$ & $257 *$ & $158 \%$ & $10.4 \%$ & $10.8 \mathrm{~ns}$ \\
\hline \multirow{4}{*}{ Cinquentinha } & C1L1 & $4.20 \% *$ & $0.83 \mathrm{~ns}$ & $0.94^{* *}$ & $14.9 \mathrm{~ns}$ & $87.4^{\% *}$ & $205 \%$ & $106 \%$ & $0.3 \mathrm{~ns}$ & $11.3 \%$ \\
\hline & C1L2 & $3.68 \%$ & $0.83 \%$ & $0.86 * *$ & $5.9 \mathrm{~ns}$ & $102.6 \%$ & $231 \%$ & $134 \%$ & $5.1 \%$ & $6.3 * *$ \\
\hline & C2L1 & $2.85^{\cdots *}$ & $0.79 \mathrm{~ns}$ & $0.84^{* *}$ & $14.9 \mathrm{~ns}$ & $94.3 \% *$ & $217 * \%$ & $125 \%$ & $0.1 \mathrm{~ns}$ & $16.7^{* *}$ \\
\hline & C2L2 & $3.19 \%$ & $0.69 *$ & $1.12 \%$ & $32.7 *$ & $89.4^{4 * *}$ & $247 * \%$ & $155 \%$ & $5.5 \mathrm{~ns}$ & $7.1 \mathrm{~ns}$ \\
\hline
\end{tabular}

${ }^{1} \mathrm{GY}$ - grain yield $\left(\mathrm{t} \mathrm{ha}^{-1}\right)$, GE - relation grain weight per ear weight, EP - ears per plant, \%DE - percentage of damaged ears, DF - days to flower, PH - plant height $(\mathrm{cm}), \mathrm{EH}$ - ear height $(\mathrm{cm}), \% \mathrm{~L}$ - percentage of root loding $(\%)$ and \%BS - percentage of stalk loding $(\%)$. $\mathrm{ns}, *$, *No significant, significant at $p \leq 0.05$ and $p \leq 0.01$, respectively.

Hallauer and Miranda Filho (1995) presented genetic correlation estimates of 0.26 and 0.31 between plant height and ear height with ear yield, respectively, on the average of 23 experiments. Estimates of $r_{A}$ between plant height and ear height with ear yield were, respectively, 0.59 and 0.51 in the base population ESALQ-PB1 (Andrade and Miranda Filho, 2008).

Nineteen estimates of $r_{A}$ between grain yield and days to flower (Table 3 ) were negatives and significant $(-0.52$ to -0.10$)$, as long as four were positive and significant $(0.43$ to 0.13$)$, suggesting that the selection of earlier maturity plants, in these maize landraces populations, could result in greater yield. Average estimate of genetic correlations across 13 populations showed that the correlation between these traits presented low value (0.14) (Hallauer and Miranda Filho, 1995).

Although the estimates of $r_{A}$ between grain yield with percentage of root loding $(\% \mathrm{~L})$ and with percentage of stalk loding (\%BS) were significant, there was no clear pattern, with the estimated values oscillating between positive and negative (Table 3).

The average of environmental $\left(\mathrm{r}_{\mathrm{E}}\right)$, additive genetic $\left(r_{A}\right)$ e phenotypic $\left(r_{F}\right)$ correlation estimates, for six landraces in the cycles and local (Table 4). For the greater part of traits a concordance in the direction and magnitude of $r_{E}, r_{A}$ e $r_{F}$ estimates were observed. In a small amount of cases that discordance existed, which can be attributed to the environmental modifier effects 
Table 3 - Estimates of the additive genetic correlation coefficients $\left(r_{A}\right)$ between grain yield (g per plant) and the other eight characters ${ }^{1}$ in different cycles $(\mathrm{C})$ and local $(\mathrm{L})$.

\begin{tabular}{|c|c|c|c|c|c|c|c|c|c|}
\hline Pop. & $\mathrm{C} / \mathrm{L}$ & GE & $\mathrm{EP}$ & $\% \mathrm{DE}$ & DF & $\mathrm{PH}$ & $\mathrm{EH}$ & $\% \mathrm{~L}$ & $\% B S$ \\
\hline \multirow{6}{*}{ Caiano } & COL1 & $0.64 * *$ & $0.69 * *$ & $-0.73 \% *$ & $-0.24 * *$ & $0.15 \%$ & $0.18 \% *$ & $0.18^{* * *}$ & - \\
\hline & COL2 & $0.58 \% *$ & $0.49 \%$ & $-0.92 \%$ & $-0.26 * *$ & $-0.09 \mathrm{~ns}$ & $0.03 \mathrm{~ns}$ & $-0.10 *$ & $0.24 * *$ \\
\hline & C1L1 & $0.42 * *$ & $0.51 \%$ & $-0.26 * *$ & $-0.16 * *$ & $0.38 * *$ & $0.33 * *$ & $0.83 * *$ & $0.75 \%$ \\
\hline & C1L2 & $0.36 \% *$ & $0.64 \% *$ & $-0.70 \% *$ & $0.05 \mathrm{~ns}$ & $0.37 \% *$ & $0.40 * *$ & - & $0.28 \% *$ \\
\hline & C2L1 & $0.23 \%$ & $0.54 \% *$ & $-0.52 \% *$ & $0.11 \mathrm{~ns}$ & $0.31 \%$ & $0.36 \%$ & $-0.30 * *$ & $0.54 * *$ \\
\hline & C2L2 & $0.80 \% *$ & $0.99 * *$ & $-0.28 * *$ & $-0.34 * *$ & $0.58 * *$ & $0.63 * x$ & - & - \\
\hline \multirow{6}{*}{ Carioca } & COL1 & $0.54 \% *$ & $0.38 \%$ & $-0.75 \% *$ & $-0.02 n s$ & $0.10 *$ & $0.094 \mathrm{~ns}$ & $0.39 \% *$ & $-0.06 n$ \\
\hline & COL2 & $0.72 \%$ & $0.97 \% *$ & $-0.45^{\% *}$ & $0.05 \mathrm{~ns}$ & $0.03 \mathrm{~ns}$ & $-0.01 n s$ & $0.02 \mathrm{~ns}$ & $0.07 \mathrm{~ns}$ \\
\hline & C1L1 & $0.31 \%$ & $0.71 \%$ & $-0.64 \% *$ & $-0.48 * *$ & $-0.04 n s$ & $-0.11 n s$ & $0.12 \%$ & $0.03 \mathrm{~ns}$ \\
\hline & C1L2 & $0.76 \% *$ & - & - & $-0.04 n s$ & $0.44 * *$ & $0.32 * *$ & $0.41 \%$ & $0.92 \%$ \\
\hline & C2L1 & $0.28 \% *$ & $0.77 * *$ & $-0.89 \%$ & $-0.34 * *$ & $0.10 \mathrm{~ns}$ & $0.09 \mathrm{~ns}$ & - & $-0.07 \mathrm{~ns}$ \\
\hline & C2L2 & $0.38 \%$ & $0.86 \%$ & - & $-0.52 * *$ & $-0.05 \mathrm{~ns}$ & $-0.21 \% *$ & - & - \\
\hline \multirow{5}{*}{ Palha Roxa } & COL1 & $0.41 \%$ & $0.39 \% *$ & $-0.39 \% *$ & $0.14 * *$ & $0.408 \%$ & $0.38 \% *$ & $0.104 *$ & $0.14 * *$ \\
\hline & COL2 & $0.91 \%$ & - & $-0.83 \% *$ & $-0.04 \mathrm{~ns}$ & $0.008 \mathrm{~ns}$ & $-0.03 \mathrm{~ns}$ & $-0.316 \%$ & $-0.13 \%$ \\
\hline & C1L1 & $0.38 \% *$ & $0.76 \%$ & $-0.95^{* *}$ & $0.28 * *$ & $0.467 * *$ & $0.45^{* *}$ & - & $0.02 \mathrm{~ns}$ \\
\hline & C1L2 & $0.34 * *$ & $0.67 * *$ & $-0.71 * *$ & $-0.06 \mathrm{~ns}$ & $0.180 * *$ & $0.13 *$ & $-0.006 n s$ & $-0.38 \% *$ \\
\hline & C2L1 & $0.28 \% *$ & $0.72 * *$ & $-0.26 * *$ & $0.07 \mathrm{~ns}$ & $0.460 * *$ & $0.39 * *$ & - & $-0.12 * *$ \\
\hline \multirow{5}{*}{ Macaco } & COL1 & $0.41 \%$ & $0.07 \mathrm{~ns}$ & $-0.61 \%$ & $0.13 * *$ & $0.26 \% *$ & $0.178 \%$ & $-0.05 n s$ & $0.08 \mathrm{~ns}$ \\
\hline & COL2 & $0.65 * *$ & $0.62 * *$ & $-0.52 * *$ & $-0.10 *$ & $0.20 * *$ & $0.228 * *$ & $-0.58 * *$ & - \\
\hline & C1L1 & $0.31 \%$ & $0.45 \%$ & $-0.55^{\% *}$ & $-0.20 * *$ & $-0.05 \mathrm{~ns}$ & $-0.033 \mathrm{~ns}$ & $-0.09 \mathrm{~ns}$ & $0.23 *$ \\
\hline & C2L1 & $0.36 * *$ & $0.87 * *$ & - & $-0.26 * *$ & $0.09 \mathrm{~ns}$ & $0.041 \mathrm{~ns}$ & $-0.59 * *$ & $0.49 * *$ \\
\hline & C2L2 & $0.47 \% *$ & $0.84 \% *$ & $-0.383 \% *$ & $-0.512 \% *$ & $0.299 \% *$ & $0.346 \% *$ & - & $0.390 \% *$ \\
\hline \multirow{4}{*}{ Maisena } & COL1 & - & $0.67 \%$ & $-0.97 \% *$ & $-0.46 * *$ & $0.29 \% *$ & $0.11 \%$ & $-0.19 * *$ & $-0.28 \%$ \\
\hline & COL2 & -- & - & $-0.92 \%$ & $-0.03 \mathrm{~ns}$ & $0.39 * *$ & $0.30 * *$ & $0.02 \mathrm{~ns}$ & $-0.27 * *$ \\
\hline & C1L1 & $0.56 * *$ & $0.65 \%$ & $-0.45 \% *$ & $-0.51 * *$ & $-0.21 * *$ & $-0.15^{* * *}$ & $-0.28 \%$ & $0.14 \% *$ \\
\hline & C1L2 & -- & $0.97 * *$ & $-0.19 * *$ & $-0.20 * *$ & $-0.04 n s$ & $-0.07 n s$ & $-0.09 \mathrm{~ns}$ & $-0.83 * *$ \\
\hline \multirow{4}{*}{ Cinquentinha } & C1L1 & $0.12 \%$ & $0.79 * *$ & $-0.82 \% *$ & $0.43 * *$ & $0.51 * *$ & $0.51^{* * *}$ & - & $0.21 * *$ \\
\hline & C1L2 & $0.61 \% *$ & $0.86 \%$ & - & $-0.09 \mathrm{~ns}$ & $0.30 * *$ & $0.37 \% *$ & $-0.13 *$ & $0.11 \%$ \\
\hline & C2L1 & -- & $0.72 * *$ & - & $0.08 \mathrm{~ns}$ & $0.02 \mathrm{~ns}$ & $0.26 \% \%$ & - & $-0.10 \mathrm{~ns}$ \\
\hline & C2L2 & $0.37 * *$ & $0.38 \% *$ & $-1.00 * \%$ & $-0.42^{* * *}$ & $0.09 \mathrm{~ns}$ & $0.15 *$ & $-0.03 \mathrm{~ns}$ & $-0.12 *$ \\
\hline
\end{tabular}

${ }^{1} \mathrm{GE}$ - relation grain weight per ear weight, EP - ears per plant, \%DE - percentage of damaged ears. DF - days to flower, PH - plant height $(\mathrm{cm}), \mathrm{EH}$ - ear height $(\mathrm{cm}), \% \mathrm{~L}$ - percentage of root loding $(\%)$ and \%BS - percentage of stalk loding (\%). ns, *, * No significant, significant at $p \leq 0.05$ and $p \leq 0.01$, respectively.

and physiologic mechanisms, which controlled the expression of the traits (Falconer and Mackay, 1996). In almost every association, the additive genetic correlations presented superior magnitudes when compared to the other types of correlation, except for the correlations of grain yield with days to flower, plant height, ear height and percentage of root loding, ears per plant with days to flower and percentage of root loding.

The average of additive genetic correlations between the trait grain yield and ears per plant was high and positive (0.67), suggesting that the selection of prolific plants resulted in a gain of selection for yield (Table 4). Similar results were shown by Lemos et al. (1992), Santos et al. (2005) and González et al. (1994). For grain yield $\times$ per- centage of damaged ears a high and negative average of additive genetic correlations $(-0.63)$ was observed, permitting the simultaneous selection of plants that have less damaged ears and bigger yields. Daros et al. (2004) reported an estimate of $r_{A}$ between grain yield $\times$ percentage of damaged equal to -0.11 , in a research with pop corn.

Days to flower presented a high positive average of additive genetic correlation with plant height $(0.56)$ and ear height (0.60). These results indicate that selection for earliness would product a reduction in plant and ear heights, in these maize landraces evaluated in family farm systems. High genetic correlations for plant height with days to anthesis (0.67) and days to silk extrusion (0.53) was evidenced by Saleh et al. (2002), in studies us- 
Table 4 - Overall average of environmental $\left(r_{F}\right)$, additive genetic $\left(r_{A}\right)$ and phenotypic $\left(r_{F}\right)$ correlation coefficients of all the maize landrace populations and the other eight characters ${ }^{1}$ (right the trace) and their standard errors of the estimates (left the trace).

\begin{tabular}{|c|c|c|c|c|c|c|c|c|c|c|}
\hline & & GY & GE & EP & $\% \mathrm{DE}$ & DF & $\mathrm{PH}$ & EH & \%L & \%BS \\
\hline \multirow{3}{*}{ GY } & $\mathrm{r}_{\mathrm{E}}$ & - & 0.39 & 0.48 & -0.35 & -0.31 & 0.23 & 0.21 & -0.08 & -0.05 \\
\hline & $\mathrm{r}_{\mathrm{A}}$ & - & 0.47 & 0.67 & -0.63 & -0.13 & 0.20 & 0.19 & -0.03 & 0.09 \\
\hline & $r_{F}$ & - & 0.40 & 0.53 & -0.43 & -0.22 & 0.21 & 0.19 & -0.09 & -0.01 \\
\hline \multirow{3}{*}{ GE } & $\mathrm{r}_{\mathrm{E}}$ & 0.11 & -- & 0.00 & -0.24 & -0.09 & 0.06 & 0.05 & -0.03 & -0.01 \\
\hline & $\mathrm{r}_{\mathrm{A}}$ & 0.19 & - & 0.18 & -0.43 & -0.27 & -0.11 & -0.06 & -0.20 & 0.19 \\
\hline & $r_{F}$ & 0.10 & -- & 0.06 & -0.29 & -0.16 & -0.01 & -0.00 & -0.05 & 0.03 \\
\hline \multirow{3}{*}{ EP } & $r_{E}$ & 0.11 & 0.05 & - & 0.02 & -0.13 & 0.08 & 0.08 & -0.03 & 0.02 \\
\hline & $\mathrm{r}_{\mathrm{A}}$ & 0.22 & 0.22 & - & -0.38 & -0.11 & 0.12 & 0.12 & 0.03 & 0.05 \\
\hline & $r_{F}$ & 0.13 & 0.08 & - & -0.09 & -0.12 & 0.10 & 0.09 & -0.05 & 0.05 \\
\hline \multirow{3}{*}{$\% \mathrm{DE}$} & $\mathrm{r}_{\mathrm{E}}$ & 0.10 & 0.14 & 0.09 & - & 0.14 & -0.09 & -0.08 & 0.02 & -0.005 \\
\hline & $\mathrm{r}_{\mathrm{A}}$ & 0.25 & 0.37 & 0.34 & - & 0.39 & 0.07 & 0.10 & 0.10 & -0.03 \\
\hline & $r_{F}$ & 0.12 & 0.17 & 0.11 & - & 0.20 & -0.03 & -0.01 & 0.04 & -0.02 \\
\hline \multirow{3}{*}{ DF } & $\mathrm{r}_{\mathrm{E}}$ & 0.11 & 0.05 & 0.09 & 0.08 & - & -0.12 & -0.11 & 0.07 & 0.01 \\
\hline & $\mathrm{r}_{\mathrm{A}}$ & 0.25 & 0.22 & 0.27 & 0.27 & -- & 0.56 & 0.59 & 0.23 & -0.11 \\
\hline & $\mathrm{r}_{\mathrm{F}}$ & 0.14 & 0.11 & 0.14 & 0.09 & - & 0.29 & 0.32 & 0.10 & -0.03 \\
\hline \multirow{3}{*}{$\mathrm{PH}$} & $\mathrm{r}_{\mathrm{E}}$ & 0.11 & 0.07 & 0.07 & 0.09 & 0.12 & - & 0.75 & -0.03 & -0.00 \\
\hline & $\mathrm{r}_{\mathrm{A}}$ & 0.21 & 0.25 & 0.22 & 0.34 & 0.16 & -- & 0.90 & 0.29 & 0.01 \\
\hline & $r_{F}$ & 0.10 & 0.09 & 0.09 & 0.09 & 0.12 & -- & 0.84 & 0.05 & 0.01 \\
\hline \multirow{3}{*}{ EH } & $\mathrm{r}_{\mathrm{E}}$ & 0.11 & 0.05 & 0.07 & 0.08 & 0.10 & 0.05 & - & -0.03 & 0.01 \\
\hline & $\mathrm{r}_{\mathrm{A}}$ & 0.21 & 0.25 & 0.22 & 0.30 & 0.17 & 0.04 & - & 0.30 & 0.10 \\
\hline & $r_{F}$ & 0.12 & 0.09 & 0.08 & 0.09 & 0.12 & 0.03 & - & 0.05 & 0.05 \\
\hline \multirow{3}{*}{$\% \mathrm{~L}$} & $r_{E}$ & 0.08 & 0.05 & 0.09 & 0.06 & 0.09 & 0.07 & 0.06 & - & -0.00 \\
\hline & $\mathrm{r}_{\mathrm{A}}$ & 0.32 & 0.23 & 0.46 & 0.37 & 0.27 & 0.27 & 0.26 & - & 0.13 \\
\hline & $r_{F}$ & 0.09 & 0.07 & 0.10 & 0.06 & 0.09 & 0.08 & 0.09 & - & 0.03 \\
\hline \multirow{3}{*}{$\% B S$} & $\mathrm{r}_{\mathrm{E}}$ & 0.08 & 0.04 & 0.11 & 0.05 & 0.05 & 0.06 & 0.06 & 0.18 & - \\
\hline & $\mathrm{r}_{\mathrm{A}}$ & 0.36 & 0.29 & 0.39 & 0.38 & 0.19 & 0.31 & 0.28 & 0.38 & - \\
\hline & $r_{F}$ & 0.07 & 0.07 & 0.13 & 0.07 & 0.07 & 0.09 & 0.09 & 0.22 & - \\
\hline
\end{tabular}

${ }^{1} \mathrm{GY}$ - grain yield (g per plant), GE - relation grain weight per ear weight, EP - ears per plant, \%DE - percentage of damaged ears, DF - days to flower, PH - plant height $(\mathrm{cm}), \mathrm{EH}$ - ear height (cm), \%L - percentage of root loding (\%) and \%BS - percentage of stalk loding $(\%)$.

ing ten corn synthetic populations. However, Santos et al. (2002) reported that phenotypic correlation between plant height and days to anthesis and days to silk extrusion, ear height and days to anthesis and days to silk extrusion, separately, were not significant.

As expected, a high average additive positive genetic correlation between plants and ears heights $(0.90)$ and a low deviation for this trait (0.04) were observed, showing a high association between these traits, over landraces and cycles of selection and local. Similar high estimates were also reported by several authors: 0.89 and 0.95 by Pinto et al. (2000); 0.84 by Silva et al. (2001); 0.78 and 0.80 by Farias Neto and Miranda Filho (2001); 0.97 by Granate et al. (2002); 0.81 and 0.82 by Santos et al. (2005).

The averages of environmental correlations were frequently small, except for the association between plant height and ear height, with coefficient average equal to 0.75 . The positive value of environmental correlation demonstrates that both characters were damaged or beneficiated by the same environmental variations and negatives values, which indicate that the environment benefited one of the characters in detriment of the other (Falconer and Mackay, 1996). The highest negative averages of environmental correlations were observed between grain yield and days to flower $(-0.31)$ and grain yield with percentage of damaged ears $(-0.35)$, indicating that the environmental conditions, that lead to increase the days to flower and damaged ears, promote a reduction of yield. This tendency can also be verified through the results showed in Table 2 .

The standard errors of the estimates of environmental, additive genetic and phenotypic correlations are 
shown in Table 4. The standard errors of the estimates of additive genetic correlations between two traits were bigger than the environmental and phenotypic correlation standard deviations, except between plant height and ear height deviation, when standard deviation of the environmental correlation was higher.

Through the correlations and their standard errors of the estimates it was possible to observe that some associations between traits always presented the same positive (grain yield with relation grain weight per ear weight and ears per plant; percentage of damaged ears with days to flower; days to flower with plant height and ear height; and, plant height with ear height) and negative sign (grain yield with percentage of damaged ears; relation grain weight per ear weight with percentage of damaged ears and days to flower).

The selection in these maize landraces to increase yield is related to the selection of plants more prolific, earlier plants, smaller percentage of damaged ears and higher relation grain weight per ear weight. There is a positive association between taller plants and increased number of days to flower, but with magnitude values that allow the selection of plants with higher yield, small height and number of days to flower. With the exception of the trait days to flower, the correlations observed in landraces within family farm systems are the same presented in several other reports (Lemos et al., 1992, González et al., 1994; Hallauer and Miranda Filho, 1995; Farias Neto and Miranda Filho, 2001; Silva et al., 2001; Saleh et al., 2002; Daros et al., 2004; Santos et al., 2005; Andrade and Miranda Filho, 2008), but with different magnitudes in some cases.

\section{Acknowledgements}

To Fundação Araucária for financial support and to AS-PTA and the family farmers that work with us.

\section{References}

Andrade, R.V.; Santos, M.X.; Ferreira, A.S.; Oliveira, A.C. 2002. Evaluation of maize land races accession collected in the Central region of Brazil. Revista Brasileira de Milho e Sorgo 1: 67-74. (in Portuguese, with abstract in English).

Andrade, J.A.C.; Miranda Filho, J.B. 2008. Quantitative variation in the tropical maize population, ESALQ-PB1. Scientia Agricola 65: 174-182.

Daros, M.; Amaral Júnior, A.T.; Pereira, M.; Santos, F.S.; Scapim, C.A.; Freitas Júnior, S.P.; Daher, R.F.; Ávila, M.R. 2004. Correlations among agronomic traits in two recurrent selection cycles in popcorn. Ciência Rural 34:1389-1394. (in Portuguese, with abstract in English).

Falconer, D.S; Mackay, T.F.C. 1996. Introduction to Quantitative Genetics. 4ed. Longmans Green, Harlow, Essex, UK.

Farias Neto, A.L.; Miranda Filho, J.B. 2001.Genetic correlation between traits in the ESALQ-PB1 maize population divergently selected for tassel size and ear height. Scientia Agricola 58: 119-123.

Ferreira, J.M.; Moreira, R.M.P.; Tardin, J.M.; Jantara, A.E. 2006. Participatory breeding landraces of maize: an experience in the Southern Central Region of Paraná State, Brazil p. 197-219. In: Labrada, H.R.; Espinosa, M.M.H.; Sotomayor, J.C.R., eds. Participatory breeding: farmers improve crop. Ediciones INCA, La Habana, Cuba. (in Spanish).
González, P.A.; Lemos, M.A.; Ramalho Neto, C.E.; Reis, O.V.; Tabosa J.N.; Tavares, Filho J.J. 1994. Genetic, phenotypic and environmental in two cycles of selection in the dent corn composite. Pesquisa Agropecuária Brasileira 29: 419-425. (in Portuguese, with abstract in English).

Granate, M.J.; Cruz, C.D.; Pacheco, C.A.P. 2002. Prediction of genetic gains from selection on popcorn half-sib families of CMS 43 population. Ciência Agrotecnologica 26: 1228-1235. (in Portuguese, with abstract in English).

Hallauer, A.R.; Miranda Filho, J.B. 1995. Quantitative Genetics in Maize Breeding. Iowa State University Press, Ames, IA, USA.

Lemos, M.A; Gama, E.E.G.; Oliveira, A.C.; Araújo, M.R.A. 1992. Correlations genotypic, phenotypic and environmental progenies of maize. Pesquisa Agropecuária Brasileira 27: 15631569. (in Portuguese, with abstract in English).

Miranda Filho, J.B. 1985. Breeding methodologies for tropical maize. p. 177-206. In: Brandolini A.; Salamini F., eds. Breeding strategies for maize production improvement in the tropics. FAO, Rome, Italy.

Nass, L.L.; Pellicano, I.J.; Valois, A.C.C. 1993. Utilization of genetic resources for maize and soybean breeding in Brazil. Brazilian Journal of Genetics 16: 983-988.

Paterniani, E. 1998. Genetic diversity and races of maize in Brazil. p. 28-31. In: Soares, A.C.; Machado A.C.; Silva B.M.; Weid J.M., eds. Maize landrace: conservation and use of biodiversity. AS-PTA, Rio de Janeiro, RJ, Brazil. (in Portuguese).

Paterniani, E.; Nass, L.L.; Santos, M.X. 2000. The value of genetic resources of maize in Brazil. p. 11-42. In: Udry C.V.; Duarte W., eds. A history of Brazilian maize: the value of genetic resources. Paralelo15, Brasília, DF, Brazil. (in Portuguese).

Pinto, R.M.C; Lima, Neto, F.P.; Souza Júnior, C.L. 2000. Estimates of appropriate number of $\mathrm{S} 1$ progenies for recurrent selection in maize. Pesquisa Agropecuária Brasileira 35: 63-73. (in Portuguese, with abstract in English).

Saleh, G.B; Alawi, S.A.S.; Panjaitan, K. 2002. Performance, correlation and herdability studies on selected sweet corn synthetic populations. Pakistan Journal of Biological Sciences 5: 251-254.

Sánchez, G.J.J.; Goodman, M.M.; Stuber, C.W. 2007. Racial diversity of maize in Brazil and adjacent areas. Maydica 52: 13-30.

Santos, P.G.; Juliatti, F.C.; Buiatti, A.L.; Hamawaki, O.T. 2002. Evaluation of the agronomic performance of corn hybrids in Uberlândia, MG. Pesquisa Agropecuária Brasileira 37: 597-602. (in Portuguese, with abstract in English).

Santos, M.F.; Moro, G.V.; Aguiar, A.M.; Souza Júnior, C.L. 2005. Responses to reciprocal recurrent selection and changes in genetic variability in IG-1 and IG-2 maize populations. Genetics and Molecular Biology 28: 781-788.

Silva, E.S.; Silva, P.S.L.; Nunes, G.H.S; Silva, K.M.B. 2001. Estimation of genetic parameters in the compound of maize ESAM-1. Caatinga 14: 43-52. (in Portuguese, with abstract in English).

Silva, H.D.; Ferreira, D.F.; Pacheco, C.A.P. 2000. Evaluation of four alternative analysis of experiments in square lattice, with the estimation of variance components. Bragantia 59: 117-123. (in Portuguese, with abstract in English).

Teixeira, F.F.; Andrade, R.V.; Oliveira, A.C.; Ferreira, A.S.; Santos, M.X. 2002. Diversity in maize germplasm collected in northeastern Brazil. Revista Brasileira de. Milho Sorgo 1: 59-67 (in Portuguese, with abstract in English).

Vencovsky, R.; Barriga, P. 1992. Genetics biometrics in plant breeding. Sociedade Brasileira de Genética, Ribeirão Preto, SP, Brazil. (in Portuguese)

Viana, J.M.S.; Regazzi, A.J. 1999. Estimation of genetic parameters in the square lattice analysis. Bragantia 58: 185-193.

Warburton, M.L.; Reif, J.C.; Frisch, M.; Bohn, M.; Bedoya, C.; Xia, X.C.; Crossa, J.; Franco, J.; Hoisington, D.; Pixley, K.; Taba, S.; Melchinger,. A.E. 2008. Genetic diversity in CIMMYT nontemperate maize germoplasma: landraces, open pollinated varieties, and inbred lines. Crop Science 48: 617-624.

Wietholter, P.; Sereno, M.J.M.S.; Terra, T.F.; Silva, S.D.A.; Barbosa Neto, J.F. 2008. Genetic variability in corn landraces from Southern Brazil. Maydica 53: 151-159.

Received December 19, 2009

Accepted January 05, 2010 